ARTICULO ORIGINAL

\title{
MADRES Y ENFERMERAS: EL MODELO DE CUIDADOS A LA INFANCIA EN LA COLECCIÓN «AL SERVICIO DE ESPAÑA Y DEL NIÑO ESPAÑ̃L» (1938-1964)
}

MOTHERS AND NURSES: THE INFANT CARE MODEL IN THE "AT THE

SERVICE OF SPAIN AND SPANISH CHILDREN” COLLECTION (1938-1964)

\section{Autores}

\author{
Salazar-Agulló, Modesta ${ }^{(*)}$ \\ Bernabeu-Mestre, Josep ${ }^{(* *)}$ \\ Ramos-Salas, Encarnación ${ }^{(* * *)}$ \\ Galiana-Sánchez, Maria Eugenia ${ }^{(* *)}$
}

(*) Departamento de Enfermería. Universidad de Alicante

(**) Departamento de Enfermería Comunitaria, Medicina Preventiva, Salud Pública e Historia de la Ciencia

${ }_{(* * *)}$ Centro de Salud Virgen del Mar. Unidad de Gestión Clínica Virgen del Mar. Almería. Servicio Andaluz de Salud.

Palabras clave: Maternidad, salud infantil, cuidados, madres, enfermeras, franquismo (1938-1964).

Keywords: Maternity, infant health, care, mothers, nurses, Francoism (1938-1964).

\section{RESUMEN}

Titulo: MADRES Y ENFERMERAS: EL MODELO DE CUIDADOS A LA INFANCIA EN LA COLECCIÓN «AL SERVICIO DE ESPAÑA Y DEL NIÑO ESPAÑOL» (1938-1964)

Objetivos: El estudio se plantea profundizar en el análisis del discurso del régimen franquista que se dirigió a la población femenina, especialmente a las madres y a las enfermeras, en relación con los cuidados a la infancia.

Métodos: Se analizó la colección «Al Servicio de España y del Niño Español» (1938-1964), 
publicada por el Ministerio de Gobernación, mediante un análisis del contenido de las monografías que se ocupaban del los cuidados a la infancia.

Discusión y conclusiones: El régimen franquista condenó el trabajo fuera del hogar de las mujeres y promovió una política pronatalista. Las mujeres fueron consideradas ignorantes y por tanto culpables de la elevada mortalidad infantil. Su acción se orientó a capacitar a todas las mujeres (que algún día llegarían a ser madres) en el cuidado de los niños. Fue obligatoria la enseñanza de la puericultura a las niñas en todos los niveles. Se fomento la lactancia materna con un discurso culpabilizador (la mujer que no lacta es una semimadre). Con todo, a pesar de todas estas iniciativas pronatalistas, la realidad social se impuso y la natalidad disminuyó, y también lo hizo la mortalidad infantil debido a la mejora de las condiciones de vida, al mismo tiempo que se produjo un fuerte aumento del uso del biberón y del trabajo femenino fuera del hogar.

\section{ABSTRACT}

Title: MOTHERS AND NURSES: THE INFANT CARE MODEL IN THE “AT THE SERVICE OF SPAIN AND SPANISH CHILDREN" COLLECTION (1938-1964)

Objectives: The aim of this study was to conduct an in-depth analysis of the discourse of the Franco regime aimed at the female population, especially mothers and nurses, regarding infant care.

Methods: A content analysis was carried out of monographs on infant care included in the collection "At the service of Spain and Spanish children" (1938-1964), published by the Home Office.

Discussion and conclusions: The Franco regime condemned the practice of women working outside the home and promoted pro-natalist policies. Women were considered ignorant and thus responsible for the high rates of infant mortality. Action was aimed at educating all women (who would one day be mothers) in child care. An education in childcare was compulsory for all girls, at all levels. Breast feeding was encouraged through a discourse of guilt (a mother who did not breastfeed was only half a mother). However, in spite of all these pro-natalist initiatives, social reality intervened and birth rates fell, as did infant mortality due to improved living conditions, whilst at the same time there was a marked increase in the practice of bottle feeding and women working outside the home.

\section{INTRODUCCION}

En sintonía con otros regímenes políticos de corte fascista, el objetivo de incrementar la población se convirtió en una las mayores preocupaciones del régimen franquista que se instauró en España al finalizar la guerra civil. Se trataba de conseguir una nación fuerte y próspera que alcanzara los 40 millones de habitantes. El problema demográfico se debía a la tendencia a la disminución de la natalidad y a las elevadas tasas de mortalidad infantil por desnutrición, asistencia sanitaria deficiente, etc. Para conseguir este objetivo se precisaba de la participación de las mujeres, en tanto que madres, así como de las profesiones más femeninas: el magisterio en todos sus ámbitos y la enfermería ${ }^{(i)}$.

Para poder alcanzar dichos objetivos, las autoridades franquistas pusieron en marcha el programa de salud materno-infantil «Al Servicio de España y del niño español». En el contexto del mismo se 
publicó una colección de monografías con el mismo título. Dicha colección se publicó entre los años 1938 y 1964. Está formada por 311 números agrupados en 252 monografías. Se trata de revistas de periodicidad mensual que estuvieron editadas por la Dirección General de Sanidad. El director de la colección y de los Servicios de Puericultura de la Dirección General de Sanidad fue el pediatra valenciano Dr. Juan Bosch Marín. Las obras estaban dirigidas a los profesionales sanitarios y estuvieron firmadas principalmente por médicos (docentes de la Escuela Nacional de Puericultura, Escuela Nacional de Sanidad, Escuelas Provinciales, facultades de medicina, etc.). Sólo un 5\% de las obras fueron firmadas por mujeres (puericultoras, matronas, doctoras en leyes, asistentes sociales, enfermeras, etc).

La colección se constituyó en el vehículo oficial de la propaganda política del régimen franquista en materia de salud materno-infantil. Los cuadernillos eran de tamaño cuartilla, escritos a doble página, y abordaron las siguientes temáticas: administración, asistencia sanitaria, demografía, propaganda y formación, salud internacional, higiene, nutrición y alimentación, principalmente ${ }^{\text {(ii). }}$

El objetivo del estudio ha sido analizar la colección «Al Servicio de España y del Niño Español» (1938-1964) y en concreto, estudiar el discurso oficial del régimen franquista que se dirigió a la población femenina, especialmente a las madres y a las enfermeras, en relación con el cuidado de la infancia.

\section{MATERIAL Y METODOS}

El estudio ha consistido en el análisis de las monografías de la colección, cuyos títulos sugieren contenidos educativos sobre el cuidado de los niños dirigidos a las madres, como Lo que deben saber las madres ${ }^{\text {(iii); }}$ Cursillo de iniciación a la puericultura: Para madres y futuras madres de la clase obrera $^{(\mathrm{iv})}$; Los padres ante la educación del niño ${ }^{(v)}$; Cuidados del niño enfermo. Errores que se cometen en su asistencia ${ }^{(v i)}$; La vida de tu nene depende de ti, mujer (vii). Además hemos enfatizado en estas monografías el análisis de los siguientes descriptores: maternidad, infancia, cuidado del niño, puericultura, lactancia, consejos.

\section{RESULTADOS Y DISCUSIÓN}

En el periodo estudiado, la iniciativa legal más ambiciosa fue la ley de Sanidad Infantil y Maternal

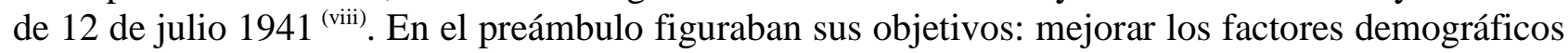
positivos estimulando la nupcialidad y natalidad y, por otro lado, combatir los factores negativos como la mortalidad de la infancia.

En la Dirección General de Sanidad, dependiente del Ministerio de la Gobernación, se concentraban las distintas secciones sanitarias que planificaban las actividades en relación con la salud maternal e infantil. La sección de Puericultura organizaba las tareas dispensariales en las clínicas de lactantes y hospitales infantiles y fueron las responsables de organizar la educación sanitaria popular mediante los cursos de enseñanza de la puericultura para madres lactantes. La sección de Maternología organizaba y dirigía las consultas de higiene prenatal y los centros maternales y pediátricos de urgencia en el medio rural, realizaba las estadísticas y controlaba la relación de asistentes a los cursos de madres lactantes. El Servicio de Propaganda publicaba la colección «Al Servicio de España y del niño español». De manera periódica daban a conocer los trabajos de divulgación sanitaria y social que consideraban de mayor interés. Editaban carteles murales de propaganda, hojas volantes de divulgación, además de una «Hoja Informativa» mensual que resumía las noticias e informaciones más destacadas, pero a las que no hemos tenido acceso. Se organizaban charlas 
radiofónicas de vulgarización de conocimientos y se promovía la enseñanza de la puericultura a las madres lactantes en todos los dispensarios de España ${ }^{(i x)}$. -

Hemos estudiado el modelo de cuidados a la infancia durante el franquismo en relación a dos aspectos fundamentales: disminuir la mortalidad infantil y aumentar la natalidad.

\section{a) Modelo de cuidados a la infancia para disminuir la mortalidad infantil}

Mientras en Europa la mortalidad infantil era de 40 niños por cada mil nacidos vivos, en España era de 112 y con grandes diferencias según la zona geográfica ${ }^{(\mathrm{x}, \mathrm{xi})}$. Las causas de ello se debían, principalmente a lo que denominaron « los tres peligros « en terminología de Mauriquand: peligro congénito, peligro alimenticio y peligro infeccioso. La cifra representaba que cada día fallecían unos 700 niños. La sensibilización sobre el problema quedaba de manifiesto en afirmaciones como la que se transcribe a continuación: «Cada día que ustedes quitan una hoja del calendario, se han perdido para España setecientas vidas de niños. ¡Setecientas madres desoladas! Setecientos hogares tristes. En la media hora que dure esta lección [...] han muerto quince niños españoles. Treinta cajitas blancas camino del cementerio en cada hora [...] Treinta cunas vacías. La mitad, por lo menos, de estas muertes, podrían evitarse y son evitables» ${ }^{(4)}$.

Las medidas propuestas para combatir este gravísimo problema fueron el fomento de la lactancia materna (peligro alimenticio), la promoción de las vacunaciones y la defensa de la higiene de la infancia (peligro infeccioso) y la promoción de los cursillos de puericultura para la educación de la mujer (peligro congénito). Se consideraba que las madres eran las verdaderas culpables, con su conducta inadecuada de cuidados a la infancia, de las elevadas cifras de mortalidad infantil. Por ello se intentó hacer obligatoria la enseñanza de la puericultura a las madres y futuras madres por Orden Ministerial de 20-12-1941, así como en las escuelas normales y en las escuelas primarias, ya que toda niña llegaría alguna vez a ser madre.

En la colección se reiteraba el mensaje de que no era la enfermedad la que mataba, sino la ignorancia. Además, la ignorancia radicaba, mucho más que en la propia madre, en las mujeres que asistían a otras en los partos, en las comadronas y en las abuelas y vecinas ${ }^{(3)}$ : «En todas estas principales causas de mortalidad, qué poco influye la miseria, o sea el estado económico; se ve que el más importante factor de la mortalidad infantil es la ignorancia, el desconocimiento de la higiene, de la crianza y de la educación de los niños. En general, nuestras mujeres no se preocupan de estos problemas, así es muy frecuente que sigan los arcaicos consejos de la abuela o los torpes conocimientos de una vecina. ¡Esto hoy no puede consentirse! [...] En pleno siglo XX, la mujer tiene que estar, en conocimientos maternales, a la altura del progreso humano y no vivir con el retraso de más de cincuenta años» (4).

Los contenidos de los cursos de puericultura eran muy similares tanto los dirigidos a las clases populares (tabla1) como los dedicados a maestras y enfermeras (tabla 2). Los de nivel más básico estaban dirigidos «a la mujer de la clase obrera, por ser la que mayor número de hijos da a la Nación, la que vive en peores condiciones higiénicas y la que desconoce en general las reglas de criar a los niños» ${ }^{(4)}$. La líder de la Sección Femenina, Pilar Primo de Rivera afirmaba que «la única tarea asignada a la mujer es su maternidad, es cuidar del hogar» (6, xii, xiii). Por tanto había que aprender a ser buenas madres. Ello significaba una separación de saberes: la madre debía saber aplicar la higiene del niño (puericultura) y el médico se ocuparía de tratar las enfermedades ${ }^{(3)}$.

La referencia a la lactancia materna era una constante. Se promovió una auténtica lucha para convencer a las mujeres sobre las bondades naturales y divinas de la lactancia materna, haciendo recaer sobre ellas la culpa si el niño no sobrevivía ${ }^{(3)}$. Consideraban la lactancia un deber de conciencia. Un axioma incuestionable era el derecho del niño a la lactancia y por tanto existía la obligación de la madre a la misma ${ }^{(6)}$. Se afirmaba que era más madre la que lactaba que la que paría 
(mater est quae lacta, non quae genuit) ${ }^{(x i v)}$. Si la mujer no lactaba lo hacía por ignorancia, por comodidad, y por tanto era una mala madre. El número de niños abandonados o expósitos era muy elevado. El principal argumento, no obstante, para lactar al neonato radicaba en que «Por cada niño que muere criado al pecho fallecen cinco criados con biberón» ${ }^{(3)}$. El fomento de la lactancia materna presente en la colección contiene un discurso no exento de cierta crueldad en ocasiones, y en general bastante culpabilizador hacia la madre. No tuvieron en cuenta las pésimas condiciones de vida de la posguerra, que hacían imprescindible que muchas mujeres se vieran obligadas a trabajar fuera del hogar para procurar la subsistencia de sus hijos.

\section{b) Modelo de cuidados a la infancia para aumentar la natalidad}

En la posguerra el número de mujeres era muy superior al de los hombres (muertos, encarcelados, fusilados o exiliados como consecuencia de la contienda). Las pautas morales del régimen constriñeron las relaciones de hombres y mujeres y las relaciones de pareja estuvieron atentamente vigiladas por una mentalidad colectiva impregnada de autocensura y de amenaza de reprobación pública, sujetas siempre a la idea de pecado, especialmente en la mujer. Se promovió un estereotipo de mujer sumisa y obediente (al padre, al esposo, al médico), dotada de manera natural para la sagrada misión que la naturaleza le había impuesto: la maternidad. Así, la mujer debía estar restringida a su ámbito natural (mujer: ángel del hogar) y por ello se combatía el trabajo femenino (xv).

Las estrategias encaminadas al aumento de la natalidad necesitaban la participación de la mujer y fueron esencialmente el fomento de la nupcialidad, la exaltación de la maternidad y la condena al uso de los métodos anticonceptivos. Existió un potente discurso ideológico para la promoción de la familia cristiana. Así la mujer española debía estar entregada en cuerpo y alma a su familia. El peor enemigo de la familia era por tanto el trabajo femenino fuera del hogar (excepción de viudas). Para capacitarlas en tan alta misión era imprescindible que aprendieran los fundamentos de la puericultura (mujer: madre y enfermera a la vez): «Le enseñaremos a las mujeres el cuidado de los hijos, porque no tiene perdón el que se mueran por ignorancia tantos niños que son siervos de Dios y futuros soldados de España» ${ }^{(x v i)}$.

Los médicos puericultores dirigieron e impartieron los cursos de puericultura en las escuelas provinciales de puericultura. Existía un férreo control de asistencia a esos cursillos, que se impartían en los primeros cinco días de cada mes y al término de los mismos les entregaban a las asistentes el diploma de MADRE EJEMPLAR (figura 1). Un extenso ejemplo de los contenidos impartidos se puede conocer en la monografía Lo que deben saber las madres, firmada por el Dr. Frías Roig, director del Instituto Provincial de Puericultura de Reus, (centro pionero en su época en la atención al niño). Se trataba de un listado de 145 consejos que comprendían todas las temáticas de puericultura, desde la higiene del embarazo, atención al parto, cuidados al recién nacido, etc. ${ }^{(3)}$. El sexismo era patente, por ejemplo, en la forma de vestir de manera diferenciada a los bebés, o en el tipo de juguetes diferenciado para niños y niñas (ya que sus objetivos pedagógicos eran diferentes): «En la niña, ante todo, la muñeca; ésta constituye un anticipo de la maternidad; encauzar este instinto es hacer puericultura, con la orientación hacia el amor, en el cariño y el hogar: características de nuestra gloriosa tradición» ${ }^{(\mathrm{xvii})}$.

Una técnica curiosa que aconsejaban se denominaba el «endurecimiento del niño». Consistía en exponerlo desnudo durante cortos periodos de tiempo para fortalecer su cuerpo ${ }^{(3)}$. Los consejos se dirigieron principalmente a las clases acomodadas ${ }^{(16)}$ ya que el médico costaba dinero. El único recurso de los pobres era acudir a las escasas consultas gratuitas de beneficencia que funcionaban en los centros rurales de higiene.

En la colección existe una condena explícita y firme al uso de los métodos anticonceptivo, incluso los naturales a pesar de que la encíclica Casti Connubii del Papa Pío xI (31 de diciembre de 1930) lo 
permitía: «hacer uso moderado del matrimonio es el único consejo que puede dar la biología a los cónyuges que con dificultad pueden sustentar una numerosa prole [...] y aprovechar para satisfacer su necesidad ciertos periodos en que la concepción es menos probable» ${ }^{\text {(xviii) }}$.

También se exhortaba a las matronas a que informaran sobre los periodos agenésicos naturales de la esterilidad femenina, ya que, para el Pontífice, era tarea de las matronas y no del sacerdote instruir a los cónyuges para difundir las normas morales ${ }^{(\mathrm{xix})}$.

Con anterioridad a la Ley de Sanidad Infantil y Maternal de 1941, el Seguro de Maternidad vigente fue el creado por Real Decreto de 22 de marzo de 1929, aunque comenzó a ser efectivo en octubre de 1931, con la II República ${ }^{\left({ }^{(x)}\right.}$. En la colección se hablaba de las excelencias de este seguro como de aquel subsidio familiar que otorgaba una serie de prestaciones a las mujeres asalariadas a las que iba dirigido (curiosamente las mismas a las que el sistema condenaba por no atender debidamente sus tareas familiares): «para la obrera, que le permite dejar el trabajo seis semanas antes y seis después del parto, con un subsidio y consulta de puericultura intrauterina, donde se hace la vigilancia médica del embarazo y se da asistencia domiciliaria del parto. Esto sin contar con las Maternidades y las Casas Maternales que, bien organizadas, tanto bien hacen en el campo de la higiene prenatal» ${ }^{(x x i, x x i i)}$.

El único tipo de familia posible era la familia cristiana y la mujer estaba obligada a transmitir estos valores a sus hijos. La protección de las familias numerosas se dirigía especialmente para las áreas rurales, (como se puede apreciar en el siguiendo texto) ya que por su condición de analfabetismo, las hacía más sensibles para la adhesión a los postulados oficiales. «Si la familia rural tiene un promedio de 5,22, la familia urbana es de 3,68. Si es verdad, como lo es, que cuanto más pobre se es, más hijos se tienen, y cuanto más ricos, menos hijos, el descenso de natalidad ha de atribuirse a causas de orden moral más que a factores de índole económica. Si ello es así, los pobres deben ser ayudados, no sólo por serlo, sino como premio al cumplimiento de sus deberes familiares» ${ }^{(18)}$. En resumen, aunque el discurso ideológico pronatalista fue muy potente, en la práctica estuvo carente de una red de recursos sanitarios y económicos.

En la divulgación de todas aquellas consignas y preceptos, tuvieron un papel destacado las enfermeras puericulturas que formaban parte del Cuerpo de Enfermeras Instructoras de Sanidad. A ellas se incorporaron las Enfermeras Visitadoras de Falange. En el ámbito rural la actuación de las enfermeras visitadoras se vio completada por las Divulgadoras Sanitarias Rurales, a quienes se encomendó la tarea educativa. La modalidad de actuación de las enfermeras fue principalmente la visita domiciliaria, en una línea de actuación muy personal « cara a cara «, daban consejos de higiene infantil y de cómo observar la buena evolución de la salud de los niños. Se las consideraba

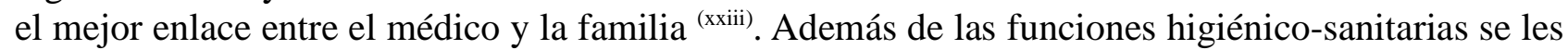
encargó de las actividades político-morales ${ }^{(x x i v)}$. Sus anotaciones, al parecer, las registraban en el Cuaderno Sanitario y en las consultas cumplimentaban un importante número de fichas de indudable valor para los registros y las estadísticas ${ }^{(\mathrm{xxv})}$.

\section{CONCLUSIONES}

Las mujeres fueron consideradas ignorantes y por tanto culpables de la elevada mortalidad infantil ${ }^{(x x v i)}$. Se intentó recluir a las mujeres al hogar, para que ejercieran el único papel en la vida: la maternidad, donde debían ejercer el papel de ángel del hogar. Fue obligatoria la enseñanza de la puericultura a todas las mujeres, maestras y enfermeras. En la colección las enfermeras y divulgadoras gozaron de cierto reconocimiento, al atribuirles el éxito de las campañas de vacunaciones infantiles y de las visitas domiciliarias realizadas en torno al nacimiento. Sin embargo, las comadronas tuvieron muy baja consideración. El planteamiento en clave de género era 
muy claro ya que se les reconocía a todas estas profesionales su idoneidad, sobre todo por su condición de mujeres.

En la colección se evidencia un fuerte tono culpabilizador y de amenaza hacia las mujeres. Como afirma Bernabeu-Mestre la mujer estaba llamada a desempeñar un papel muy activo en la lucha contra la mortalidad infantil, tan importante o más como el que se le atribuía en la empresa de remontar la natalidad y que se resumía en: «[...] criar al pecho a sus hijos mientras no hubiere causa justificada que lo impidiese, esforzarse en conocer y practicar las reglas de la puericultura, solicitar de forma inmediata la asistencia médica, y de forma particular en el caso de las diarreas y por último, vacunar a los hijos contra la tuberculosis, la viruela, la difteria y la fiebre tifoidea» ${ }^{(x x v i i)}$.

La realidad social se impuso a los principios programáticos que se difundieron a través de la colección «Al Servicio de España y del niño español». La natalidad no solo no aumentó, sino que disminuyó, lo que también actuó a favor de una mejor salud de los niños. Como también apunta Beatriz Echeverri, a partir de 1955 se produjo un repunte de la fecundidad de las españolas pero sólo entre las mujeres jóvenes, lo que indica que, al alcanzar el número deseado de hijos, las parejas ponían los medios necesarios para controlar su fecundidad, a pesar de que la venta de anticonceptivos estaba prohibida en España ${ }^{(x x v i i i)}$.

La mortalidad infantil disminuyó hasta unas tasas próximas al 42 por mil pero fue debido más a las

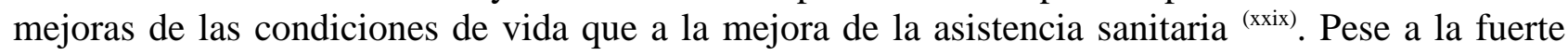
condena del uso del biberón, se produjo un incremento de éste. La crianza de los hijos se flexibilizó y se produjo la incorporación femenina al mercado laboral de manera irreversible. 
Tabla 1. Cursillos de Puericultura dirigidos a las mujeres y madres lactantes ${ }^{(4)}$

\begin{tabular}{|l|l|}
\hline Lección primera & ¿Por qué mueren tantos niños en España? ¿Qué es la puericultura? \\
\hline Lección segunda & Higiene del embarazo. Atenciones al recién nacido. Cuidados al niño enfermo. \\
\hline Lección tercera & Lactancia materna, artificial y mixta. Sus ventajas e inconvenientes. \\
\hline Lección cuarta & Destete. Alimentación del niño hasta los tres años. Cocina dietética infantil. \\
\hline Lección quinta & Enfermedades infecciosas más frecuentes en la infancia. Cómo se evita la tuberculosis. \\
\hline Lección sexta & Higiene de la boca y la garganta. Lo que no se debe hacer con los niños. \\
\hline Lección séptima & La escuela y el niño. Guarderías infantiles. Jardines de la infancia. \\
\hline Lección octava. & La moral en el niño. Instituciones de puericultura. La mujer y el niño en la Nueva España \\
\hline
\end{tabular}

Tabla 2. Cursillos de formación puericultora para enfermeras y maestras ( ${ }^{\mathrm{xx}}$ )

- 30 temas de higiene infantil

- 15 alimentación

- 7 epidemiología

- 15 maternología

- 11 higiene especial

- 6 higiene preescolar

- 18 higiene escolar

- 12 prácticas de laboratorio

- 6 prácticas de psicología infantil

- 7 prácticas en consulta

- 1 práctica de radiología

- 6 prácticas en guarderías

Figura 1. Diploma de madre Ejemplar ${ }^{(4)}$

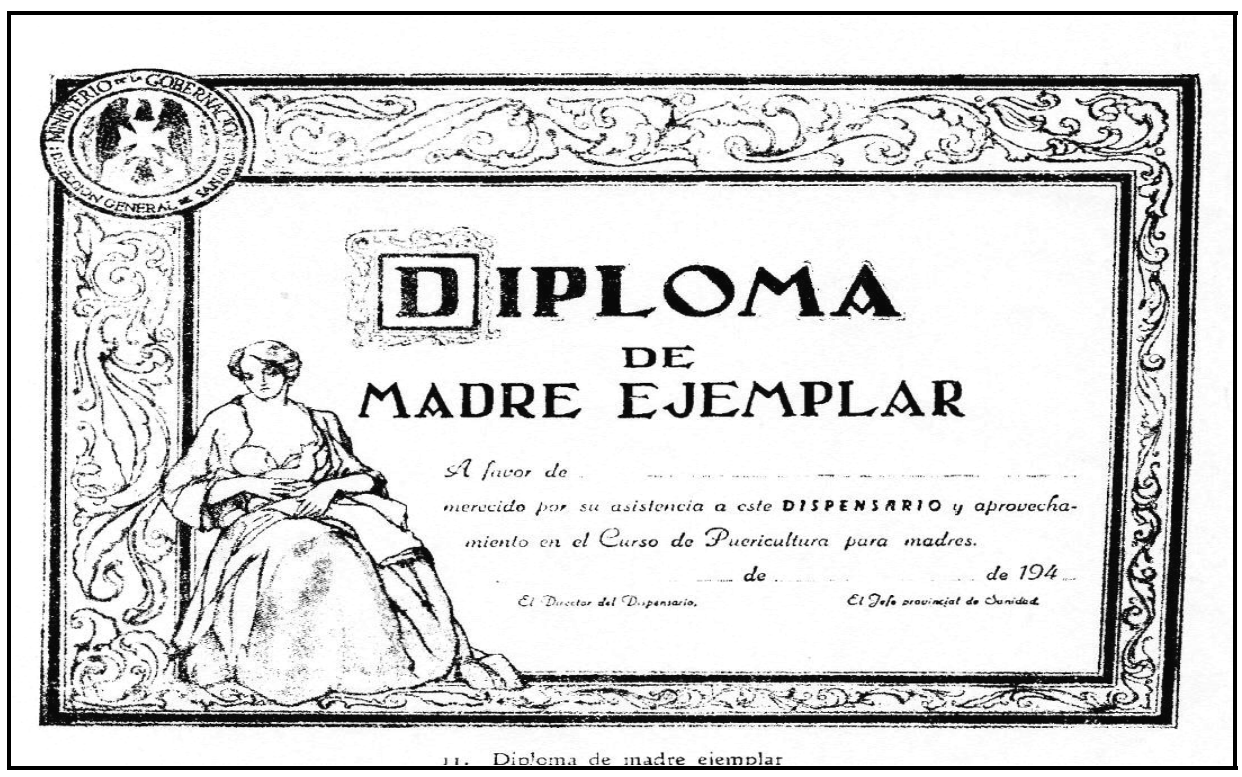




\section{FUENTES Y BIBLIOGRAFIA}

Fernández-Salinero De Miguel C, Rabazas Romero T. La orientación vocacional de las mujeres en el Franquismo. En: XIII Coloquio Internacional de la Asociación Española de Investigación de Historia de las Mujeres. Barcelona, 19-21 de octubre, Edición CDRom, 2006.

Salazar-Agulló M, Martínez-Marco EA, Bernabeu-Mestre J, La salud materno-infantil durante el primer franquismo: Notas bibliométricas sobre el Programa "Al Servicio de España y del Niño Español). Asclepio, Rev. Hist. De la medicina y de la Ciencia, 2007, vol. LVIX, $\mathrm{n}^{\mathrm{o}} 1$, enero-junio.

Frías Roig Alejandro, (1946), Lo que deben saber las madres, Madrid, Ministerio de la

Gobernación, septiembre, (Al Servicio de España y del Niño Español, No 103).

González-Álvarez, Matutina (1939), Cursillo de iniciación a la puericultura: Para madres y futuras madres de la clase obrera, Madrid, Ministerio de la Gobernación, noviembrediciembre, (Al Servicio de España y del Niño Español, No 23-24: 6-7).

Isern y Galcerán Carmen, (1959), Los padres ante la educación del niño, Madrid, Ministerio de la Gobernación, noviembre, (Al Servicio de España y del Niño Español, N ${ }^{\mathbf{0}}$ 261).

Martínez Andrés (1940), Cuidados del niño enfermo. Errores que se cometen en su asistencia, Madrid, Ministerio de la Gobernación, enero, (Al Servicio de España y del Niño Español, $\left.\mathrm{N}^{\circ} 25\right)$.

Mestre Medina Joaquín, (1939), La vida de tu nene depende de ti, mujer, Madrid, Ministerio de la Gobernación, febrero, (Al Servicio de España y del Niño Español, N ${ }^{\circ}$ 14).

Comité de Redacción, (1941), Ley de Sanidad Infantil y Maternal, de 12 de julio de 1941, s.l., Ministerio de la Gobernación, agosto (Al Servicio de España y del Niño Español, 42).

Bravo Sánchez del Peral Enrique, (1950), La sanidad infantil y maternal en España: Su organización actual, servicios que comprende, legislación vigente, s.l., Madrid, Ministerio de la Gobernación, noviembre-diciembre, (Al Servicio de España y del Niño Español, 153-154).

Bosch Marín Juan, (1947), El niño español en el siglo XX, Madrid, Instituto de España, Real Academia Nacional de Medicina, marzo-junio, (Al Servicio de España y del Niño Español, 109-110-111-112).

Bernabeu-Mestre Josep, Caballero Pablo, Galiana-Sánchez $M^{a}$ Eugenia, Nolasco Andreu (2006), Niveles de vida y salud en la España del primer franquismo: las desigualdades en la mortalidad infantil, Rev. Dem. Hist., XXIV, I y II: 173-193.

Villar Salinas Jesús, (1942), La natalidad contemporánea en España, Madrid, Ministerio de la Gobernación, mayo-junio, (Al Servicio de España y del Niño Español, 51-52).

Richmond K, Las mujeres en el fascismo español. La sección femenina de la falange, 1935-1959. Madrid, Alianza Editorial, 2004.

Morales y González Juan Luis, (1951), La lactancia materna es ineludible deber de conciencia y, desde el punto de vista sanitario, un grave delito su abandono. El papel del médico católico en su defensa, Sevilla, Editorial Católica Española, S.A., (Al Servicio de España y del Niño Español, 164).

Pérez-Fuentes Hernández Pilar, (2000), El trabajo de las mujeres: una mirada desde la historia, Lan Harremanak, 2000 (1): 185-209.

Bosch Marín Juan, (1940), Política familiar y sanitaria, s.l., Ministerio de la 
Gobernación, mayo, (Al Servicio de España y del Niño Español, 27).

Ruiz Santamaría Juan A, (1945), Semana del niño en Valencia. Primera exposición de higiene infantil, s.l., Ministerio de la Gobernación, enero, (Al Servicio de España y del Niño Español, 83).

Bosch Marín Juan, (1938), Problemas de Maternología y Puericultura, Valladolid: imprenta provincial, Ministerio de la Gobernación, marzo, (Al Servicio de España y del Niño Español, 3).

Comité de Redacción (1952), Moral y relaciones conyugales, Madrid, Ministerio de la Gobernación, marzo, (Al Servicio de España y del Niño Español, 169: 22-23).

Martínez Veiga Ubaldo, (1995), Mujer, trabajo y domicilio. Los orígenes de la discriminación, Icaria Ed., S.A., Barcelona.

Álvarez Romero Enrique, (1939), Higiene infantil e instructoras de sanidad, Valladolid, Ministerio de la Gobernación, enero, (Al Servicio de España y del Niño Español, 13).

Giménez y González-Claramunt Vicente, (1942), Las mutualidades maternales, s.l, Ministerio de la Gobernación, agosto, (Al Servicio de España y del Niño Español, 54).

Galiana-Sánchez $\mathbf{M}^{\mathrm{a}}$ Eugenia, Bernabeu-Mestre Josep, Alimentación, enfermería y cultura: el antecedente histórico de las visitadoras puericultoras. En: Alimentación, Salud y Cultura: encuentros interdisciplinares, IX Coloquio Redam, Tarragona (en prensa).

Galiana Sánchez Ma Eugenia, Bernabeu-Mestre Josep, García-Paramio Pilar, Enfermeras para una nueva patria: género e ideología en la enfermería de Falange. En Gónzalez Canalejo Carmen (ed.). Historia de la Enfermería. Desde las sociedades ágrafas hasta la contemporaneidad, Almería, Universidad de Almería (en prensa).

Bernabeu-Mestre Josep, Perdiguero Enrique, Barona Josep Lluis, (2007), Determinanti della mortalità infantile e transizione sanitaria. Una riflessione a partire dall'esperienza espagnola. En Breschi, Marco, Pozzi, Lucia, Salute, Malattia e Sopravvivenza in Italia fra '800 e '900, Udine: Forum: 175-193.

Palacio Lis Irene, (2003), Consejos a las madres: Autoridad, ciencia e ideología en la construcción social de la función materna. Una mirada al pasado, Rev. Sarmiento, 7: 6180 .

Bernabeu-Mestre, Josep (2002), Madres y enfermeras. Demografía y salud en la política poblacionista del primer franquismo, 1939-1950, Rev. Dem. Hca., xx, I, 2002, segunda época.

Echeverri Dávila, Beatriz, (2003), La protección de la infancia: la Educación Sanitaria de las madres en la posguerra española, Historia y Política: Ideas, procesos y movimientos sociales, 9: 279-308.

Gómez Redondo, Rosa (1985), El descenso de la mortalidad infantil en Madrid, 19001970. REIS 32/85: 101-139.

Comité de Redacción (1948), Programas de puericultura, maternología e higiene escolar en 1948, s.l., Ministerio de la Gobernación, Dirección General de Sanidad, Sección de Puericultura, Maternología e Higiene escolar, marzo, (Al Servicio de España y del Niño Español, 121). 